\title{
Major disruption of the pelvic ring during normal vaginal delivery: A case report
}

\author{
$M$ van Heukelum MBChB, DA(SA) \\ C Blake MBChB, MMed(Ortho)UFS
}

Department of Orthopaedic Surgery, Worcester Provincial Hospital, Western Cape, South Africa

\author{
Corresponding author: \\ Dr Marcus van Heukelum \\ Department of Orthopaedic Surgery \\ Worcester Provincial Hospital \\ Western Cape, South Africa \\ Tel: +27023348 1100 \\ Email: marcusvanh@gmx.com
}

\begin{abstract}
Open-book pelvic fractures are generally associated with high energy trauma. Occasionally these fractures are seen as a complication of normal vaginal delivery. We report the case of a 25-year-old female with a $77 \mathrm{~mm}$ symphysis pubis diastasis with associated disruption of the sacroiliac joints following delivery, which was successfully treated with open reduction and internal fixation.
\end{abstract}

Key words: pubic symphysis diastasis, pregnancy, McRoberts, fundal pressure, operative management pelvis fracture

http:/ / dx.doi.org/10.17159/2309-8309/2017/v16n1a2

\section{Background}

Pubic symphysis diastasis during vaginal delivery is a rare and frequently undiagnosed complication. ${ }^{1}$ The reported incidence varies widely, ranging from $1 / 569$ to $1 / 30000$ deliveries. ${ }^{1-5}$ Diastasis may occur during the antepartum, intrapartum and postpartum periods.

The degree of pubic symphyseal diastasis varies considerably between individuals. Physiological widening is minor and generally asymptomatic. Separation exceeding $10 \mathrm{~mm}$ is generally symptomatic, associated with pain and difficulty weight bearing, and is considered pathological. ${ }^{1,4,6,7}$ Separation of the pubic symphysis exceeding $25 \mathrm{~mm}$ leads to progressive injury of the posterior pelvic ring, including sacroiliac joint disruption or sacral fracture., ${ }^{4,6}$

The bones and ligaments of the pelvis adapt remarkably in pregnancy, allowing significant pelvic laxity. This increase in mobility of the sacroiliac, sacrococcygeal and pubic joints are considered normal and are necessary for childbirth. Complex interactions between relaxin, progesterone and oestrogen lead to relaxation of the connective tissue of the ligaments, allowing the joints to respond to the mechanical stress of childbirth. ${ }^{1,6,8}$ The exact relationship between these hormones and joint laxity remains unclear. ${ }^{8}$ These responses begin to regress immediately after delivery and regression is complete by 6 months.
Increases in peripheral-joint laxity during pregnancy do not correlate with serum relaxin, oestrogen or progesterone levels. ${ }^{6,8}$

In this environment of increased pelvic joint mobility, delivery manoeuvres such as anterior fundal pressure and the McRoberts manoeuvre may increase the risk of symphyseal diastasis. ${ }^{79}$

\section{Case presentation}

We present a case of major pubic symphysis diastasis with sacroiliac disruption in a healthy multigravida after fullterm, vaginal delivery.

A 25-year-old woman (gravida: 3, para: 3, $157 \mathrm{~cm}$, $71 \mathrm{~kg}, \mathrm{BMI}=28$ ) with no known comorbidities and an uncomplicated prenatal course was referred to our orthopaedic department with pelvic pain and an inability to weight bear after normal vaginal delivery. She was referred by a local day hospital after delivering a healthy baby boy $(4400 \mathrm{~g}, 62 \mathrm{~cm}, 35 \mathrm{~cm}$ head circumference). The spontaneous delivery occurred at 39 weeks' gestation and was conducted by a trained nursing sister. No instrumentation or medication was used to induce or augment labour and no complications were reported.

The patient reported that uterine fundal pressure had been applied and that her hips had been placed in a flexion/abduction 'McRoberts' position during delivery. 
She reported severe right-sided hip pain shortly after delivery and was unable to weight bear.

Examination revealed a haemodynamically stable patient with pain over the pubic symphysis as well as sacroiliac joints; the pain radiated down the right leg. The pubic symphysis was clinically displaced with localised swelling. Gynaecological examination revealed no marked bleeding and was unremarkable apart from swelling over the vulva. Her abdomen was soft and non-tender and she passed clear urine without difficulty. Lower limb neurovascular examination was normal.

Anterior-posterior radiograph of the pelvis showed an open-book pelvis with $77 \mathrm{~mm}$ pubic symphysis diastasis plus disruption of the sacroiliac joints (Figure 1). Injury was in keeping with a Young and Burgess AP compression type II (Figure 2). The pelvis was assessed as being unstable.

We opted to stabilise the pelvic ring, and surgical fixation was performed with open reduction and internal fixation. A horizontal 'Pfannenstiel' incision was used, the pubic symphysis was reduced, clamped, and a six-hole small fragment reconstruction plate was placed across the superior pubic rami. Laparoscopic tubal ligation was performed at the same setting.

Post-operative management included: bed rest for four weeks, analgesia, thromboprophylaxis with compression stockings plus IM Clexane (40 IU daily) and in-bed physiotherapy. The patient developed superficial surgical site sepsis which resolved after washout in theatre plus IV Cloxacillin (1g 6 hrly) for seven days.

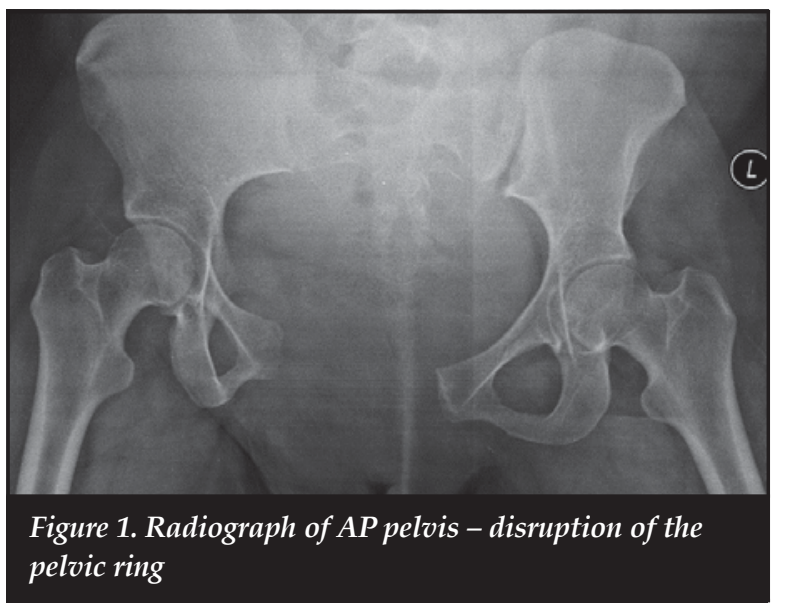

She was discharged four weeks post-surgery, she was able to fully weight bear and mobilised without aid; the surgical wound had healed well.

At the six-week follow-up, she had symphyseal pain but mobilised independently without aid. Radiographs confirmed reduction of the pubis symphysis and sacroiliac joints (Figure 3).

At the three-month follow-up the patient had mild pain over the symphyseal region but was mobilising well; the reduction had maintained.

We plan to monitor the patient and are currently not planning implant removal.

\section{Discussion}

There are many case series or case reports discussing pregnancy-related pubic symphysis diastasis; most deal with minor disruption and are treated non-operatively. In this case, we report a severe $(77 \mathrm{~mm})$ symptomatic symphysis diastasis with posterior pelvic arch instability. This degree of pelvic disturbance is usually associated with high energy trauma, typically Young and Burgess anterior-posterior (AP) compression-type injuries. ${ }^{10}$

In pregnancy-associated symptomatic diastasis, the mean gap is $20 \mathrm{~mm}$ (range: $10-35 \mathrm{~mm}$ ), the sacroiliac joint becomes affected when separation exceeds $40 \mathrm{~mm}$. In extreme cases, separation as much as $120 \mathrm{~mm}$ has been reported. ${ }^{6}$

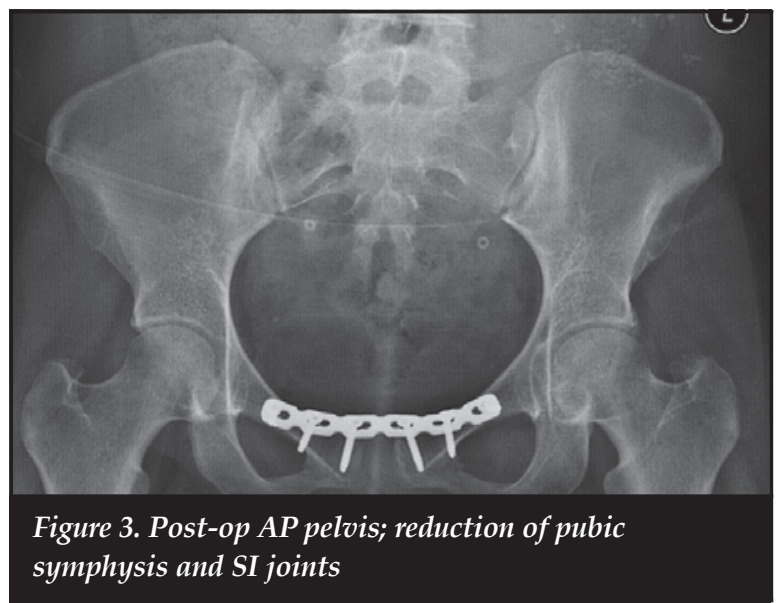

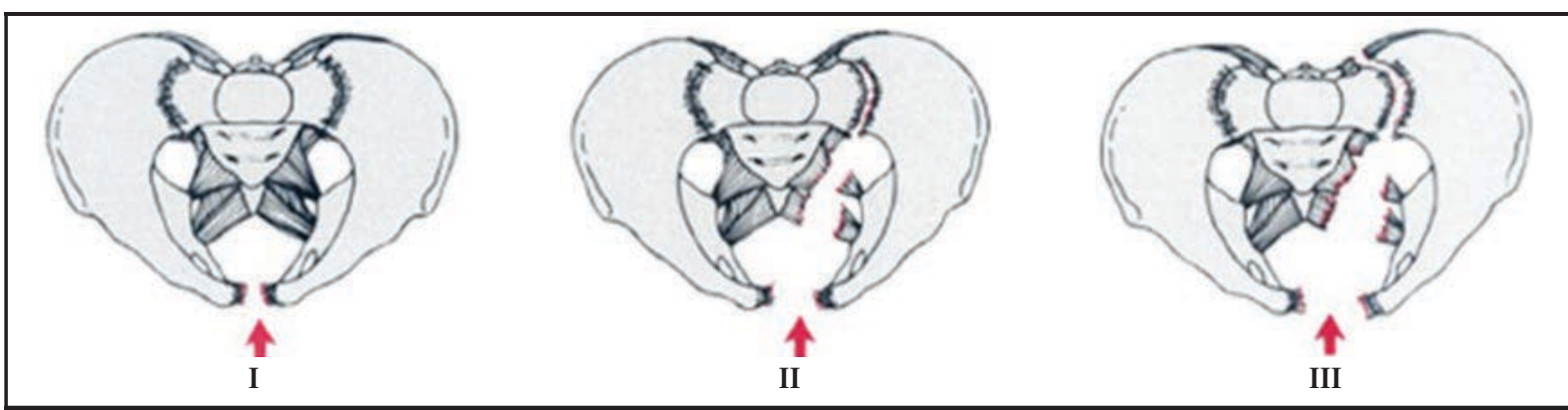

Figure 2. Young and Burgess AP compression-type fractures 
AP compression injury, classified by Young and Burgess into three types (Figure 2), results from an anteriorly directed force applied directly to the pelvis or indirectly via the lower extremities. Type II injuries are typically associated with high energy; $>2.5 \mathrm{~cm}$ of anterior ring diastasis with opening of the sacroiliac joints resulting in rotational instability. The posterior sacroiliac ligaments remain intact. These injuries are often associated with neurovascular injuries, soft tissue complications and haemorrhage. They usually require close observation and aggressive resuscitation. ${ }^{10}$

The aetiology of symptomatic diastasis associated with normal vaginal delivery is not fully understood. Numerous potential risk factors have been suggested, including: precipitous labour, cephalopelvic disproportion, macrosomia, shoulder dystocia, multiparity, previous trauma or excessive force applied to the pelvic ring, excessive abduction of the thighs during delivery, instrument-assisted delivery, pre-existing abnormalities due to congenital dysplasia, osteomalacia, chondromalacia or rickets and excessive hormone-related softening of the ligaments during pregnancy. ${ }^{1,5,6,11}$

In our case, the patient reported the use of uterine fundal pressure as well as the McRoberts position during delivery. Uterine fundal pressure aims to reduce the duration of the second stage of labour. This manoeuvre is widely used although no confirmed benefit has been documented and adverse events have been reported. The McRoberts manoeuvre, which is generally safe, may result in diastasis, especially when excessive force is used or when used for prolonged periods. ${ }^{7}$ We believe that in our case, the use of inappropriate and uncontrolled force in the above-mentioned manoeuvres may have contributed to the diastasis resulting in a severe pelvic injury, usually associated with high energy trauma.

The incidence of symptomatic separation appears to be decreasing, as many difficult vaginal deliveries and operative instrumental deliveries are increasingly replaced by Caesarean section. ${ }^{1}$

Most cases of symphysis pubis diastasis following vaginal birth can be successfully managed non-operatively with bed rest, analgesia, activity restriction, pelvic binders and physiotherapy.,12 Non-operative treatment usually results in rapid alleviation of symptoms and complete recovery in $4-8$ weeks. ${ }^{1,5,11}$

Cases where diastasis exceeds $40 \mathrm{~mm}$, indicating posterior pelvic arch instability, require reduction and stable fixation. ${ }^{11}$ External fixation or internal fixation of the superior pubic rami with plate and screw or cerclage wire is the treatment of choice. This provides stability while the ligaments heal. ${ }^{13,14}$ Additional percutaneous screw fixation of the sacroiliac joint is a treatment option for synchronous symphyseal and sacroiliac joint disruption. ${ }^{4}$

Surgical treatment is also indicated when non-operative treatment fails. Failure includes: inadequate reduction, recurrent diastasis or persistent symptoms. ${ }^{11,13,15}$
Surgical fixation of severe symphysis separation allows for earlier ambulation and ability to care for self and baby. ${ }^{15}$ Injuries require 6-12 weeks to heal; we mobilised our patient at four weeks post-surgery as she was painfree and her social circumstances as well high risk for thrombosis necessitated earlier mobilisation.

Patients with unstable pelvic disruption following delivery should be managed in the same manner as trauma patients, with vigilant haemodynamic monitoring, aggressive resuscitation and timely operative reduction and fixation of the pelvis.

Although internal fixation of the symphysis pubis is commonly performed, there are no clear guidelines regarding the indications for implant removal. The longterm effects of retaining these internal fixation devices are not well described. Complications include: anterior symphysis pain, sexual dysfunction, infection and implant failure.

There is no consensus in the literature regarding implant retention and removal after internal fixation of the symphysis pubis. The current recommendation is that implant removal should be considered on an individual basis depending on the presence of local symptoms which may be attributed to the retention of the implant. ${ }^{16}$

The risk of symphyseal diastasis recurrence in subsequent pregnancies is over $50 \%$; risk factors remain poorly defined. The mode of delivery for subsequent pregnancies should be carefully discussed with the mother but risk of recurrence should not alter the management of these

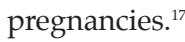

\section{Conclusion}

Parturition-associated disruption of the pelvic ring is a rare complication. Pregnancy-associated physiological changes together with the use of the outdated McRoberts manoeuvre and the application of fundal pressure can lead to symphyseal diastasis. We postulate that the use of inappropriate force during the McRoberts manoeuvre may have contributed to the severe pelvic injury in this case.

We opted to manage a severe pelvic ring injury with open reduction and internal fixation with good outcomes.

\section{Compliance with Ethics Guidelines}

The authors declare that there is no conflict of interest regarding the publication of this paper.

\section{Reference}

1. Lindsey RW, Leggon RE, Wright DG, Nolasco DR. Separation of the symphysis pubis in association with childbearing. A case report. JBJS Case Connector. $1988 \mathrm{Feb}$ 1;2:289-92.

2. Taylor RN, Sonson RD. Separation of the pubic symphysis. An underrecognised peripartum complication. The Journal of reproductive medicine. 1986 Mar;31(3):203-206 
3. Dhar S, Anderton J. Rupture of the symphysis pubis during labor. Clinical Orthopaedics and Related Research. 1992;\&NA;283:252-57.

4. Hierholzer C, Ali A, Toro-Arbelaez JB, Suk M, Helfet DL. Traumatic disruption of pubis symphysis with accompanying posterior pelvic injury after natural childbirth. American Journal of Orthopedics (Belle Mead, NJ). 2007 Nov;36(11):E167.

5. Snow R, Neubert A. Peripartum pubic symphysis separation. Obstetrical \& Gynecological Survey. 1997;52(7):438-43.

6. Çıçek H, Keskın H, Tuhanığlu Ü, Kiliçarslan K, Oğur H. Simultaneous disruption of the pubic symphysis and sacroiliac joint during vaginal birth. Case Reports in Orthopedics. 2015;2015:1-5.

7. Joosoph J, Kwek K. Symphysis pubis diastasis after normal vaginal birth: a case report. Annals of the Academy of Medicine, Singapore. 2007 Jan;36(1):83-85.

8. Marnach M, Ramin K, Ramsey P, Song S, Stensland J, An $\mathrm{K}$. Characterization of the relationship between joint laxity and maternal hormones in pregnancy. Obstetrics $\mathcal{E}$ Gynecology. 2003;101(2):331-35.

9. Api O, Emeksiz Balcin M, Ugurel V, Api M, Turan C, Unal $\mathrm{O}$. The effect of uterine fundal pressure on the duration of the second stage of labor: A randomized controlled trial. Acta Obstetricia et Gynecologica Scandinavica. 2009;88(3):320-24.

10. Guthrie H, Owens R, Bircher M. Fractures of the pelvis. Journal of Bone and Joint Surgery - British Volume. 2010;92B(11):1481-88.
11. Kharrazi F, Rodgers W, Kennedy J, Lhowe D. Parturitioninduced pelvic dislocation: a report of four cases. Journal of Orthopaedic Trauma. 1997;11(4):277-81.

12. Jain N, Sternberg L. Symphyseal Separation. Obstetrics $\mathcal{E}$ Gynecology. 2005;105:1229-32.

13. Chang J, Wu V. External fixation of pubic symphysis diastasis from postpartum trauma. Orthopedics. 2008;31(5):1-3.

14. Rommens P. Internal fixation in postpartum symphysis pubis rupture: report of three cases. Journal of Orthopaedic Trauma. 1997;11(4):273-76.

15. Dunivan G, Hickman A, Connolly A. Severe separation of the pubic symphysis and prompt orthopedic surgical intervention. Obstetrics \& Gynecology. 2009;114:473-75.

16. Raman R, Roberts C, Pape H, Giannoudis P. Implant retention and removal after internal fixation of the symphysis pubis. Injury. 2005;36(7):827-31.

17. Gillaux C, Eboue C, Herlicoviez M, Dreyfus M. Antécédent de disjonction pubienne et modalités d'accouchement. Journal de Gynécologie Obstétrique et Biologie de la Reproduction. 2011;40(1):73-76.

This article is also available online on the SAOA website (www.saoa.org.za) and the SciELO website (www.scielo.org.za). Follow the directions on the Contents page of this journal to access it. 